Archives de sciences sociales des religions

125 | janvier - mars 2004

Autorités religieuses en islam

\title{
Musulmans indo-pakistanais et autorité religieuse en diaspora : le cas américain
}

Aminah Mohammad-Arif

\section{(2) OpenEdition}

Édition électronique

URL : http://journals.openedition.org/assr/1041

DOI : 10.4000/assr. 1041

ISSN : $1777-5825$

Éditeur

Éditions de l'EHESS

Édition imprimée

Date de publication : 1 janvier 2004

Pagination : 147-163

ISBN : 2-222-96741-4

ISSN : 0335-5985

Référence électronique

Aminah Mohammad-Arif, « Musulmans indo-pakistanais et autorité religieuse en diaspora : le cas américain », Archives de sciences sociales des religions [En ligne], 125 I janvier - mars 2004, mis en ligne le 22 février 2007, consulté le 19 avril 2019. URL : http://journals.openedition.org/assr/1041 ; DOI : 10.4000/assr.1041 


\section{MUSULMANS INDO-PAKISTANAIS ET AUTORITÉ RELIGIEUSE EN DIASPORA : LE CAS AMÉRICAIN}

\section{Introduction}

Depuis les années 1960, un nombre croissant de musulmans sud-asiatiques (1), parmi lesquels une proportion significative de personnes au niveau d'instruction élevé, a choisi l'Amérique comme terre d'immigration. Ils se sont montrés particulièrement actifs et dynamiques dans l'institutionnalisation de l'islam aux États-Unis. Mais, comme à tant d'autres, s'est posée à eux la question du leadership religieux. Celui-ci s'avère crucial en immigration à un double titre : il conditionne la perpétuation même de la religion islamique en contexte migratoire ; il est indispensable dans la négociation avec les autorités locales.

Aux États-Unis, la question revêt un aspect particulier en raison du statut spécial conféré au religieux : si les Américains ont établi une séparation entre le religieux et le politique (Establishment Clause), le premier amendement à la constitution n'en garantit pas moins la liberté religieuse (Freedom of Religion Clause). Toutefois, en laissant entendre que l'État ne peut donner une primauté au religieux sur le non-religieux, ni favoriser une tradition religieuse au détriment d'une autre, la constitution protège l'égalité des citoyens au sein de la nation, mais laisse subsister une ambiguïté quant à la nature des rapports entre l'Église et l'État. La religion est théoriquement reléguée à la sphère privée mais les relations entre le civil et le religieux restent équivoques. Le ciment nationaliste lui-même repose sur la notion de religion civique " qui n'empiète aucunement sur les religions particulières mais qui, au contraire, s'appuie à l'occasion sur elles, et les renforce à son tour » (2). La population elle-même se caractérise par le plus fort taux d'adhésion religieuse des pays industrialisés. Ce phénomène s'accompagne d'une tradition de

(1) Les musulmans sud-asiatiques incluent ici les Indiens, les Pakistanais et les Bangladeshis. On estime le nombre de musulmans sud-asiatiques aux États-Unis à plus de 800000 sur un total de musulmans variant entre 4 et 6 millions selon les estimations. Ils sont concentrés dans un nombre relativement restreint d'États : la Californie, l'État de New York, le New Jersey, l'Illinois et le Texas.

(2) Elise Marienstras, « Nation et religion aux États-Unis », in Patrick Michel, dir., Religion et démocratie, Paris, Albin Michel, 1997, p. 278. 
tolérance religieuse, héritée de l'histoire même des États-Unis, lesquels ont été créés par des minorités religieuses qui fuyaient l'oppression. Cette tolérance a permis aux musulmans de pratiquer leur religion et de la revendiquer de façon relativement ouverte. Surtout, spécificité toute américaine, elle a laissé le champ libre, ou presque, aux leaders religieux auto-proclamés, musulmans et non musulmans, et créé les conditions d'une compétition, parfois acharnée, entre et au sein même des groupes religieux.

Depuis les attentats du 11 septembre 2001, les Américains considèrent cependant les musulmans avec une certaine suspicion. D'où, pour ces derniers, la nécessité d'offrir de leur communauté une image rassurante, notamment par la voie de leurs leaders. Cet impératif, devenu capital, vital même, pour leur survie en tant que minorité jouissant pleinement de sa liberté religieuse, couplé aux exigences d'adaptation en situation diasporique et à l'ancienneté croissante de la présence islamique sur le territoire américain, ont quelque peu transformé la physionomie du leadership religieux aux États-Unis.

La scène musulmane américaine se définit avant tout par sa grande fragmentation. Aux États-Unis, les musulmans représentent en effet un microcosme de la population islamique mondiale, tant y sont divers et nombreux les groupes et sous-groupes ethniques, linguistiques, sectaires (3), idéologiques, etc. Les lieux de culte reflètent cet éclatement de la scène religieuse. Bien qu'aucune mosquée ne déclare officiellement représenter un groupe ethnique particulier, la réalité est tout autre, les mosquées tendant à être largement établies par affiliation ethnico-nationale. Celle-ci se lit en particulier à l'origine ethnique de l'imam et à la composition du conseil d'administration de la mosquée. En l'absence d'une autorité religieuse « établie »- placée sous le contrôle de l'État ou d'un groupe d'oulémas dominants -, ce contexte éminemment pluriel laisse une large place à la concurrence. Hormis les Sud-Asiatiques, trois groupes ethniques aspirent à asseoir leur légitimité : les Arabes, les Afro-Américains, les Blancs convertis (même si ces derniers ne représentent qu'un très faible pourcentage du total des musulmans aux États-Unis). Forts de leur «bagage » historique et de leur accès direct aux Textes, les Arabes tendent à considérer qu'ils détiennent le monopole de la légitimité religieuse et à se voir comme «naturellement» musulmans. Les Afro-Américains arguent de l'ancienneté de leur présence aux États-Unis qui, en leur octroyant la qualité «d'autochtones », ferait d'eux des « experts » de la société américaine, sans compter le rôle qu'ils ont joué dans l'implantation des premières structures religieuses destinées aux musulmans, dont tous les immigrants, y compris ceux d'Asie du Sud, ont bénéficié lorsque eux-mêmes n'avaient pas encore établi leurs propres mosquées. Enfin, Afro-Américains et Blancs convertis jouent un rôle intellectuel non négligeable dans la définition et l'orientation de l'islam aux États-Unis : ainsi, ils portent un regard désapprobateur sur le bagage culturel et ethnique des imams arabes ou indo-pakistanais et sur leur usage de l'autorité religieuse. Face à ces trois groupes, les Sud-Asiatiques cherchent leur place. Ils se montrent même particulièrement dynamiques dans ce marché concurrentiel, puisqu'ils ont joué un rôle crucial dans la création de nombre des institutions islamiques américaines les plus notables (la Muslim Student Association (MSA), l'Islamic Society of North America (ISNA), l'Islamic Circle of North America (ICNA), etc.). Rappelons ici

(3) Nous ne nous intéresserons ici qu'aux sunnites. 
brièvement que la MSA a été fondée en 1963 par des étudiants musulmans (dont un certain nombre étaient originaires du sous-continent indien) qui s'étaient fixé comme objectifs d'aider les étudiants dans leur pratique quotidienne, d'informer les non-musulmans sur l'islam afin d'écarter les préjugés et de se livrer, plus ou moins directement, à des activités de prosélytisme. Dans le prolongement des activités de la MSA, deux organisations, à caractère non exclusivement estudiantin, mais désireuses de viser plus largement les immigrants, ont vu le jour, en 1971 et en 1981. Il s'agit respectivement de l'ICNA qui regroupe essentiellement des Indo-Pakistanais, et de l'ISNA, une organisation-fédérative et trans-ethnique (4).

Mais cette concurrence n'exclut pas les contacts entre groupes ethniques (en particulier entre Arabes et Sud-Asiatiques amenés à travailler ensemble sinon dans les mosquées du moins dans les organisations islamiques), pas plus qu'elle ne suggère une homogénéisation au sein de ces groupes, eux-mêmes traversés par des courants divers (notamment en matière d'autorité religieuse). Il ne faut pas non plus surestimer cette concurrence à bases ethniques car, qu'ils soient ou non immigrants, les musulmans tendent encore à se tourner de préférence vers leur propre groupe ethnico-national pour toute consultation en matière religieuse.

Par ailleurs, si, dans leurs pays d'origine, les musulmans sud-asiatiques n'avaient certes pas d'autorité religieuse unanimement reconnue comme telle, ils disposaient néanmoins, à chaque coin de rue ou presque, d'une pléthore d'oulémas et de soufis en tous genres. Tel n'est plus le cas aux États-Unis où ces immigrants sont en quête d'autorités religieuses susceptibles de leur fournir des repères, de leur dire ce qu'est la norme, alors qu'eux-mêmes sont souvent désorientés par la situation diasporique.

Nous commencerons par exposer la représentation que les immigrants ont de leur imam; puis, nous verrons quelles sont les sources possibles d'autorité religieuse des musulmans indo-pakistanais aux États-Unis ainsi que leur évolution éventuelle, en nous concentrant sur le cas de New York; enfin, nous examinerons le lien entre l'autorité religieuse et le politique.

\section{Les immigrants et leurs imams : attentes et préoccupations}

Traditionnellement, les vertus qu'attendent les croyants d'un leader religieux musulman sont les suivantes : une ascendance ashraf (noble) (5), la piété, le savoir, l'orthodoxie, l'intégrité personnelle, la probité morale, la générosité, etc. (6) Plus

(4) Aminah Mohammad-Arif, "Ilyâs et Maudûdî au pays des yankees », Archives de Sciences Sociales des Religions, $\mathrm{n}^{\mathrm{o}} 117,2002$, pp. 175-203.

(5) Cela sous-entend dans le sous-continent indien une descendance du Prophète, de ses compagnons, ou encore plus largement d'Arabes, d'Iraniens, ou de Turcs (par opposition aux convertis locaux).

(6) Cf. notamment Simon Digby, "The Sufi Shaikh as a Source of Authority in Mediaeval India », in Marc Gaborieau, dir., Islam et société en Asie du Sud, Paris, Éditions de 1'EHESS, 1986, pp. 60-62 (coll. «Purushartha ») ; Usha SANYal, Devotional Islam and Politics in British India: Ahmad Riza Khan Barelwi and his Movement, 1870-1920, Delhi, OUP, 1996, pp. 128-166. 
généralement, «des individus et des institutions se voient investis d'une autorité parce qu'on estime qu'ils incarnent et exemplifient l'ordre moral » (7). Ces qualités attendues ont évolué dans le monde musulman, et plus encore sans doute en diaspora. Aux États-Unis, elles demeurent bien entendu fondamentales (sauf peut-être l'ascendance qui ne semble pas revêtir une dimension aussi capitale en diaspora), mais elles ne suffisent plus. D'autres qualifications deviennent tout aussi, voire plus, importantes encore aux yeux des fidèles: outre une solide formation en sciences religieuses, les compétences linguistiques (connaître et l'arabe et l'anglais), une bonne connaissance de la société américaine, ainsi que la capacité à bien saisir les implications d'une vie en minorité, y compris chez les Indiens (pourtant déjà minoritaires en Inde, mais jouissant d'un statut particulier à maints égards) (8). L'aptitude à transmettre un enseignement vivant et "parlant» aux enfants constitue également une autre des qualifications requises. Alors que dans le sous-continent indien l'instruction, religieuse en particulier, tend encore à privilégier la mémorisation des textes, sans effort critique, les enfants scolarisés dans les écoles américaines connaissent un système d'éducation qui privilégie le questionnement. Aussi, les parents recherchent-ils pour leurs enfants des enseignants capables de communiquer avec eux de façon compréhensible et attrayante. Ils craignent aussi que des imams «importés » (9) n'inculquent à leurs enfants des valeurs qui les incitent à l'isolationnisme. Cette appréhension est symptomatique du tiraillement des immigrants entre le souci de transmettre à leurs enfants leur héritage religieux et leur volonté de voir ces derniers s'intégrer dans la société américaine (10).

À toutes ces qualités s'ajoute la capacité à négocier efficacement avec les autorités locales et à projeter en direction de la société d'accueil, une image positive de la communauté. Moyennant quoi, la légitimation se construit autant par la faculté à communiquer avec l'autre, que par le fait que la société d'accueil considère tel leader comme un interlocuteur acceptable. L'autorité religieuse s'établit donc non seulement par rapport à la communauté elle-même, mais aussi par rapport à la société (et à l'État) d'accueil. Or, depuis le 11 septembre, ce dernier aspect a acquis une dimension particulière, les activités des musulmans, en tant que groupe religieux, faisant l'objet d'une étroite surveillance de la part des autorités américaines.

La communication avec l'autre s'opère également par le truchement de dialogues interreligieux dont une partie des Américains, associés aux Églises protestantes et catholiques libérales (par opposition aux (ultra)-conservateurs de droite), sont particulièrement friands. Ces dialogues entre musulmans et non musulmans se sont multipliés après le 11 septembre car ils constituent eux aussi un moyen d'intégration et d'acceptation par une société américaine qui, au quotidien, valorise le religieux. Or, ces dialogues nécessitent la participation de personnes « ouvertes » à l'autre et suffisamment familiarisées avec le contexte américain.

(7) Dale F. Eikelman, James Piscatori, Muslim Politics, Delhi, OUP, 1997, p. 58.

(8) Les musulmans indiens disposent par exemple de leur propre code civil.

(9) Voir ci-dessous.

(10) C'est pourquoi la vaste majorité des parents sud-asiatiques n'envoie pas ses enfants dans les écoles islamiques à plein temps, et préfère se contenter des cours du dimanche à la mosquée. Cf. Aminah Mohammad-Arif, Salam America, L'islam indien en diaspora, Paris, CNRS Éditions, 2000 (coll. "Monde indien. Sciences sociales $15^{\mathrm{e}}-20^{\mathrm{e}}$ siècle ») (cf. Arch. 114.31). 
Nombre des imams «importés» du sous-continent indien n'étant pas en mesure de répondre à ces attentes et à ces préoccupations, d'autres personnes, au moins parmi les Sud-Asiatiques, se chargent d'assumer ces rôles multiples.

\section{L'imam de la mosquée : entre auto-proclamation et importation}

L'absence d'autorités religieuses durant les premières années de l'immigration des musulmans aux États-Unis a favorisé l'émergence de leaders autoproclamés qui, du fait de cette auto-désignation, ne disposaient que d'une légitimité très relative. En effet, lorsque les premières mosquées ont été fondées en Amérique, la charge de l'imam était fréquemment assumée par des novices et/ou des autodidactes qui n'avaient reçu aucune formation islamique. Ces novices tendaient à imposer des points de vue plus ou moins fantaisistes, dont la conformité avec l'orthodoxie pouvait être mise en doute, ce qui eut pour effet de ne guère inciter les fidèles de l'époque à fréquenter les mosquées.

Cette première phase de l'histoire des musulmans aux États-Unis a été suivie par une deuxième (qui subsiste aujourd'hui encore à bien des égards), celle des imams « importés ». Pour combler ce déficit en autorité religieuse, les immigrants, sud-asiatiques notamment, arrivés massivement à partir des années soixante, ont fait venir des pays musulmans ou à fortes minorités musulmanes, comme l'Inde, des imams qui ont bénéficié d'une formation traditionnelle et qui prêchent et/ou dispensent un savoir plus conforme à l'orthodoxie que leurs prédécesseurs. Pour autant, «l'importation » d'imams «correctement» formés à la loi islamique ne constitue pas la solution idéale en terre d'immigration. En effet, ces imams ne possèdent généralement qu'une connaissance très limitée de la société américaine et ne sont guère aptes à répondre aux attentes d'immigrants dont la priorité est l'intégration, même s'ils cherchent parallèlement à préserver leur identité musulmane. Il en résulte chez ces derniers, et plus encore chez leurs enfants, un sentiment de désaffection vis-à-vis des institutions islamiques en place. Or, la transmission de l'héritage religieux demeure l'une des préoccupations majeures des immigrants originaires du sous-continent indien. Par ailleurs, si traditionnellement, dans les pays musulmans, la reconnaissance de l'autorité par les pairs est au moins aussi essentielle que la reconnaissance par les fidèles, en terre d'immigration, la légitimation par ces derniers revêt probablement un caractère encore plus important. Car ce sont les fidèles qui « sponsorisent» les figures religieuses : celles-ci sont quasiment à leur merci, les immigrants pouvant les embaucher et les congédier à leur guise. Cela signifie que même si elles inspirent encore un certain respect, elles ont quelque peu perdu de leur «autorité » en diaspora. Ce système qui n'accorde guère de protection aux imams offre au contraire aux immigrants une assez large marge de manœuvre. Il est symptomatique de la liberté dont jouissent les musulmans dans le champ religieux américain.

Certains de ces imams sont rétribués par des pays étrangers. Ce qui implique que leurs prêches doivent satisfaire les intérêts de ces derniers (et réduit d'autant leur marge de liberté). La question de l'autonomie du leadership religieux en 
immigration se pose aussi du fait que nombre de mosquées aux États-Unis sont financées par des pays étrangers: pays du Golfe, Pakistan, Bangladesh. Or, le contexte de l'après 11 septembre est propice à la remise en question d'une telle dépendance envers des pays étrangers. Toutefois, bien avant ces événements déjà, un certain nombre de mosquées, attachées à leur liberté, s'étaient, dès leur établissement, prononcées en faveur d'une totale autonomie, y compris financière, vis-à-vis de toute tutelle étrangère. Tel a été notamment le cas de l'Islamic Center of Long Island (ICLI), situé dans une banlieue aisée de New York, essentiellement dominé par des Sud-Asiatiques. Cette autonomie financière dont jouissent certaines mosquées aux États-Unis, comme l'ICLI, n'est possible que dans un contexte de succès économique et financier, lequel est lui aussi symptomatique de l'appartenance sociale de nombre d'immigrants musulmans aux États-Unis, d'origine sud-asiatique en particulier. Dans la mesure où ils disposent de moyens financiers conséquents, ces derniers en effet peuvent " s'offrir le luxe » d'une double distanciation : vis-à-vis des tutelles étrangères, et vis-à-vis des banques auprès desquelles ils n'ont pas à contracter d'emprunts. Ce faisant, il se mettent également en conformité vis-à-vis de l'interdiction islamique sur le prêt à intérêt.

Le cas de l'ICLI présente aussi un intérêt en raison de sa façon de « gérer » son imam. Rappelons d'abord que dans nombre de mosquées des États-Unis, comme la "grande mosquée » de Manhattan (ou Islamic Cultural Center of New York) financée par des pays islamiques, la fonction de l'imam s'est considérablement transformée et élargie. Elle tend à s'apparenter à celle des prêtres et des rabbins. Loin de se cantonner à diriger la prière et à délivrer des sermons l'imam assume bien d'autres tâches : il émet des avis sur des questions de croyances et de pratiques religieuses et il participe au fonctionnement administratif de la mosquée. À la fois enseignant et conseiller matrimonial, il rend visite aux malades dans les hôpitaux et veille au bon déroulement des cérémonies funèbres. Lorsqu'il maîtrise l'anglais, il peut aussi devenir le porte-parole de la communauté auprès de la société d'accueil, et informer les Américains sur l'islam en prononçant des discours dans les églises, les synagogues ou les écoles (depuis le 11 septembre cette dernière fonction est devenue très importante). En devenant ainsi un "représentant» de la mosquée tout entière, sa sphère d'autorité s'élargit d'autant.

Dans les pays musulmans, on observe rarement ce cumul des fonctions, nombre des tâches dévolues à l'imam en immigration y étant assumées par des qấlî (11), des muftî (12), etc. Ce phénomène, également observable en Europe, doit son émergence au contexte diasporique et à la pénurie en autorité religieuse qui en résulte. Pourtant, aux États-Unis, toutes les mosquées ne suivent pas ce schéma, tant s'en faut, en particulier lorsqu'elles sont dominées par des Sud-Asiatiques.

Ainsi, à l'ICLI, l'un des centres islamiques les mieux organisés de New York, l'imam est loin de concentrer en ses mains la totalité du pouvoir. Certes, il a été " importé » du Pakistan et sa formation à Médine et à al-Azhar lui a conféré une certaine légitimité aux yeux des fidèles de la mosquée. C'est d'ailleurs grâce à ces qualités qu'il doit d'avoir été embauché. Mais il ne cumule guère les fonctions puisqu'à son strict rôle d'imam menant la prière ne s'ajoute que celui de " gardien du temple» : logé avec sa femme et ses enfants dans une maisonnette située dans

(11) Juge.

(12) Juriste. 
l'enceinte même du centre islamique, il est en effet chargé de garder les lieux. Son prédécesseur, qui professait une vue étroite de l'islam et souhaitait que seules ses opinions fassent autorité, fut congédié par le conseil d'administration et remplacé par le présent imam dont les fonctions ont été strictement définies et limitées dès le départ. Même le sermon n'est pas de son ressort exclusif, d'autres membres de l'ICLI se chargeant à l'occasion de le prononcer (selon les préoccupations du jour). L'imam peut néanmoins être sollicité pour répondre à des questions d'ordre religieux; mais les autres tâches, tout aussi essentielles, tant pour garantir le bon fonctionnement du centre islamique que pour asseoir la légitimité de l'ICLI aux yeux des fidèles, sont assurées par des personnes différentes.

L'ICLI s'est doté d'une constitution en vertu de laquelle tous les deux ans, le comité exécutif est réélu, de même que trois membres du conseil d'administration. C'est à ce dernier, démocratiquement élu, que revient la tâche de gérer la mosquée et de choisir l'imam. Le conseil d'administration a d'autre part créé des comités en tous genres. Chacun d'eux est présidé par des personnes différentes, hommes ou femmes, membres du centre et bénéficiant dans leur grande majorité d'un niveau d'éducation élevé. La plupart sont des comités d'aide qui répondent aux divers problèmes des immigrants (sans lien direct nécessairement avec le religieux) : organisation de la rupture du jeûne, arrangement des funérailles, relations dans le couple et lutte contre la violence domestique, aide à la recherche d'un emploi, etc. Certains comités ont une fonction relationnelle : prise de contact avec les autres mosquées, mise en relation avec les églises et les synagogues pour établir des dialogues interreligieux, etc. Un comité spécial est chargé des relations avec la presse. Une fois encore, l'imam de cette mosquée, dont la connaissance de l'anglais est plutôt sommaire, a été écarté de cette fonction. Cette dernière est assumée par deux membres de l'ICLI qui ne possèdent pas de formation en sciences religieuses, mais qui sont hautement éduqués, maîtrisent parfaitement l'anglais (même s'ils sont eux-mêmes des immigrants) et se targuent d'une bonne compréhension de la société américaine grâce à une installation dans le pays vieille de plus de 30 ans.

Enfin, l'instruction religieuse des enfants est une autre des fonctions qui échappent au contrôle de l'imam. On l'a vu, les parents attachent une très grande importance à la transmission de l'islam à leur progéniture. Aussi se sont-ils mis en quête d'enseignants américains. Au départ, les méthodes d'éducation empruntaient largement à celles en vigueur dans le sous-continent, mais les enfants ne manifestaient qu'un enthousiasme modéré face à des cours ennuyeux et répétitifs. Les responsables de l'ICLI changèrent alors leurs méthodes, s'inspirant plus largement des méthodes américaines qui accordent une place plus large à la réflexion et à la participation des élèves en classe. Ils engagèrent des musulmans américains, convertis pour la plupart, plus rodés aux techniques d'enseignement à l'américaine.

Il est remarquable que d'autres mosquées dirigées par des Sud-Asiatiques, qui ne possèdent pas nécessairement le même degré d'organisation que l'ICLI, tendent à fonctionner sur ce modèle. L'imam y assume un minimum de fonctions, le reste étant assuré par d'autres membres généralement plus éduqués et donc supposés plus compétents. Dans la ville de New York, il en est ainsi par exemple de l'autre grande mosquée dirigée par des Indo-Pakistanais, le Muslim Center of New York. Le même scénario est observable dans une mosquée de Manhattan fréquentée essentiellement par des chauffeurs de taxi sud-asiatiques et dont l'imam n'est jamais une personne fixe mais un homme différent désigné chaque semaine par le 
Directeur de la mosquée pour mener la prière. Ce dernier est pour sa part un comptable de profession. Il a opéré ce choix de diriger une mosquée afin de poursuivre une tradition familiale : son propre grand-père administrait une mosquée en Inde et son père un lieu de prière à Hong-kong. Ce cas de figure constitue un exemple (peu fréquent du reste) d'un leadership constitué dans le prolongement d'une tradition familiale.

De toutes les mosquées sud-asiatiques visitées à New York, la seule où l'imam cumule les fonctions est une mosquée bangladeshi : la formation et la personnalité de son imam le distinguent des autres de ses collègues rencontrés. Il est en effet le plus éduqué d'entre eux : ancien professeur d'études islamiques à l'université de Dacca, il maîtrise parfaitement, outre le bengali, l'anglais, l'arabe et l'ourdou. Grâce à son niveau d'éducation élevé, non seulement cet imam exerce plusieurs fonctions (il est enseignant, conseiller matrimonial, porte-parole de la mosquée à l'extérieur, etc.) mais il remplit également le rôle de coordinateur de toutes les mosquées bangladeshi de New York.

Hormis cet exemple assez exceptionnel chez les Sud-Asiatiques, dans les autres cas observés, on l'a dit, la plupart des imams se bornent à diriger la prière, les autres fonctions nécessaires au bon fonctionnement d'une mosquée ou d'un centre islamique étant généralement assumées par des immigrants ne possédant aucun « background » religieux, ni de par leur ascendance familiale, ni même de par leur « compétence » ou leur formation en matière religieuse. Les images traditionnellement accolées au mollah (13) dans le sous-continent - objet fréquent de dérision, voire de mépris, perçu comme " borné », "stupide », " hypocrite » - expliquent en partie ce phénomène car ces préjugés tendent à être véhiculés en immigration. Soulignons ici le contraste entre Sud-Asiatiques et Afro-Américains. Chez ces derniers, probablement influencés par leurs congénères protestants, dont les pasteurs sont réputés pour leur charisme, nombreux sont les personnages religieux charismatiques, comme l'imam Siraj Wahhaj de la mosquée al-Taqwa de Brooklyn, dont les discours ne manquent pas de soulever l'enthousiasme des foules. Les musulmans noirs américains confèrent donc une grande importance au charisme de leur leader. Rien de tel n'est observable chez les Sud-Asiatiques aux États-Unis (14). En tout état de cause, ils ne semblent pas compter dans leurs rangs de leaders dont le charisme personnel suffirait à asseoir leur légitimité (15).

En l'absence de personnages charismatiques notables et parce qu'ils éprouvent des difficultés à se défaire de l'image peu flatteuse du mollah, les Indo-Pakistanais sont plutôt les partisans d'un partage des pouvoirs. Nombre de mosquées, comme l'ICLI, ont un fonctionnement démocratique, grâce, on l'a vu, à un système d'élections régulières du conseil d'administration. Celui-ci partage le pouvoir avec l'imam et les participants assidus aux activités des mosquées. Ces derniers, en versant une partie de leur salaire à l'institution islamique de leur choix, aident non

(13) Mollah est l'équivalent persan du terme arabe mawla. Il est utilisé dans le sous-continent pour désigner (souvent péjorativement) un 'alim au sens large, celui-ci pouvant tout aussi bien être un imam qu'un muftî, etc.

(14) Dans le sous-continent en revanche, il existe diverses figures religieuses qui jouissent d'un certain charisme auprès de la population (chefs d'organisations islamiques, shaikhs soufis, etc.).

(15) Notons simplement l'existence d'un soufi originaire de Sri Lanka, Bawa Muhaiyaddeen (mort en 1986), qui a établi un sanctuaire en Pennsylvanie. Mais il ne comptait que très peu de disciples chez les Sud-Asiatiques peu portés au soufisme aux États-Unis, pour des raisons expliquées ailleurs. cf. Aminah Mohammad-Arif, Salam America, op.cit. 
seulement au financement de celle-ci, mais s'arrogent également le droit d'influer sur sa politique. C'est donc bien une autorité religieuse singulière qui se développe autour de la mosquée en contexte américain.

Le niveau élevé d'éducation de la plupart des Indo-Pakistanais les conforte dans leur volonté de partager les pouvoirs, et les rend particulièrement attentifs au contenu des sermons et enseignements délivrés dans les mosquées. Il convient cependant de noter que ce niveau d'instruction n'encourage pas nécessairement les immigrants à adopter des vues progressistes. C'est même l'inverse qui se produit : fragilisés par la situation migratoire ils ont plutôt tendance à embrasser des positions conservatrices en matière de mariage, de divorce ou d'homosexualité. Sur ces questions, leurs vues ne seront pas très éloignées de celles d'un imam directement importé de l'Inde ou du Pakistan. Néanmoins, alors qu'en Inde comme au Pakistan les autorités religieuses sont connues pour être particulièrement rétives au changement (16), aux États-Unis la plupart des immigrants perçoivent les nécessités d'adaptation imposées par le contexte diasporique et voient d'un œil critique les discours sclérosés de leurs imams importés. Ainsi, la plupart des Sud-Asiatiques accordent une grande importance à l'instruction laïque de leurs filles, alors que certains des imams importés, héritiers d'une vision archaïque, sont hostiles à une inscription trop visible des femmes musulmanes dans la sphère publique américaine subséquente à la poursuite d'études supérieures.

Quoi qu'il en soit, la situation des Indo-Pakistanais se distingue sur au moins un point encore de celles des Afro-Américains : ces derniers n'éprouvent guère de difficultés à se sentir américains et à ce titre s'estiment plus libres (parce qu'ils sont chez eux) de prononcer des discours enflammés. Les Sud-Asiatiques, en revanche, se sentent étrangers aux États-Unis (en particulier lorsqu'ils appartiennent à la première génération), tout en aspirant, pour la plupart, à être intégrés. C'est pourquoi ils concentrent tous leurs efforts pour être acceptés par la société-hôte. Soucieux de renvoyer d'eux-mêmes une image positive aux Américains, dès avant le 11 septembre, ils ne toléraient pas dans leurs mosquées des figures religieuses dont les discours trop critiques envers la société américaine auraient découragé l'intégration aux États-Unis. Tout en éprouvant la nécessité d'avoir des imams traditionnels, ne serait-ce que pour diriger la prière, car eux-mêmes ne possèdent pas de formation religieuse adéquate, les membres (tous immigrants dans la plupart des cas) du conseil d'administration des mosquées dirigées par les Sud-Asiatiques œuvrent de manière à n'accorder à ces derniers qu'un pouvoir limité et à exercer sur eux un (étroit) contrôle. Depuis le 11 septembre les immigrants n'ont fait que redoubler de vigilance vis-à-vis des discours de leurs imams.

On ne saurait toutefois négliger la part de l'ambition personnelle dans ces questions de partage des pouvoirs. En d'autres termes, le contrôle des imams et la volonté de s'assurer que ces derniers n'exercent qu'une autorité limitée ne sont pas exempts d'enjeux de pouvoir et de rivalités internes entre les immigrants pour les positions de leadership au sein des mosquées et autres institutions islamiques. D'autant que dans la plupart des cas, y compris pour des immigrants éduqués et qui ont socialement réussi, ces institutions (auxquelles on peut ajouter les associations communautaires culturelles et politiques) sont souvent les seules instances où ils

(16) Philip Lewis, Islamic Britain: Religion, Politics and Identity among British Muslims, Londres, I.B Tauris Publishers, 1994, pp. 131-134. 
peuvent espérer exercer un certain pouvoir. Très minoritaires et surtout (trop) récemment établis aux États-Unis, ils ne disposent de guère d'autres vecteurs d'expression.

Néanmoins des voix critiques s'élèvent régulièrement et s'interrogent sur la légitimité de leaders d'institutions islamiques sans formation religieuse. De là est née l'idée de constituer des séminaires et des centres de formation islamique aux États-Unis même.

\section{Les alternatives à l'imam}

\section{Formation locale}

Afin de répondre aux difficultés soulevées par la présence d'imams importés, voire catapultés par des instances étrangères, les musulmans aux États-Unis ont tenté de trouver d'autres solutions, au nombre desquelles la création en 1983 à Chicago de l'American Islamic College (AIC). Cette institution, dont plusieurs des enseignants sont des Indo-Pakistanais, dispense un enseignement visant à la formation, sur le sol américain, d'imams ou tout au moins d'une élite musulmane. Cependant, face à l'incapacité à rénover l'enseignement islamique, cette tentative s'est trouvée en état d'échec total. Les immigrants musulmans, particulièrement les Indo-Pakistanais, soucieux, en outre, de l'intégration de leurs enfants dans la société américaine, préfèrent les envoyer dans des institutions de l'enseignement supérieur laïques.

Plutôt que de faire appel à un imam pour les questions relatives au droit islamique, les musulmans américains se sont progressivement tournés vers des « experts » locaux. Bien qu'ils ne possèdent pas nécessairement toutes les qualifications traditionnellement requises d'un muftî, ces derniers ont une bonne connaissance de la société américaine à laquelle s'ajoute une formation en arabe et en études islamiques, ce qui leur confère une légitimité suffisante aux yeux des fidèles qui, pour certains d'entre eux, se tourneront volontiers vers ces néo-muftîs (17) pour solliciter leur opinion en matière religieuse.

L'islam suscite un intérêt croissant aux États-Unis. Ce qui a eu pour effet d'inciter certains spécialistes musulmans de l'islam à poursuivre leurs études supérieures aux États-Unis, voire à y enseigner, tandis que, de leur côté, un nombre grandissant de musulmans américains, dont des Indo-Pakistanais, s'engagent dans des études islamiques aux États-Unis, et/ou partent à l'étranger pour y recevoir une instruction religieuse dans des universités et des institutions islamiques (18). Parmi ces derniers on compte un nombre grandissant de jeunes.

(17) Mon expression.

(18) Yusuf Talal Delorenzo, «The Fiqh Councilor in North America », in Yvonne Haddad, John Esposito, eds., Muslims on the Americanization Path?, New York, OUP, 2000, p. 67. 


\section{Organisations nationales}

Les immigrants, en quête d'une autorité capable de les informer sur leur religion et de les aider dans leur pratique quotidienne, ont également la possibilité de se tourner vers des organisations comme l'Islamic Society of North America (ISNA) et l'Islamic Circle of North America (ICNA) (19). L'une comme l'autre sont à l'écoute des immigrants, grâce notamment à des services téléphoniques gratuits (toll-free hotlines) que les fidèles peuvent appeler à tout moment, vingt-quatre heures sur vingt-quatre, pour poser des questions relatives à la pratique quotidienne, au comportement à adopter en telle ou telle circonstance, etc. Les personnes chargées de leur répondre n'ont pas toutes une formation classique de 'alim, mais elles sont engagées pour «la cause de l'islam » depuis de longues années : la plupart des membres de l'ICNA, largement dominé par les IndoPakistanais, étaient avant d'émigrer, et/ou sont encore, des militants de la Jama'at-i Islami (20), donc familiarisés avec les enseignements de son fondateur, Abul A'la Maudûdî, lequel représente à leurs yeux une source majeure d'autorité religieuse. Parmi ceux qui se mettent ainsi au service des croyants peuvent aussi se trouver des oulémas au sens plus classique du terme : l'ICNA, par exemple, a son propre 'alim, en la personne de Maulânâ (21) Naseem, un musulman indien émigré aux États-Unis en 1980. Il a été formé à l'Université d'Aligarh, où il a étudié la psychologie et la philosophie, et à la Nadwa't-ul 'Ulamâ (22), où il a appris l'arabe et le droit islamique.

L'ICNA et l'ISNA organisent des congrès annuels dans lesquels sont débattus des sujets couvrant des questions relatives à la condition des femmes, à la violence domestique, à l'éducation des enfants, au prosélytisme, et depuis peu au 11 septembre et à ses implications pour les musulmans. L'ISNA se préoccupe également de la formation des imams puisque, depuis 2001, elle organise des conférences annuelles sur ce sujet (annual imam training seminar).

À l'initiative de l'ISNA, de l'ICNA et d'autres organisations islamiques a également été créé le Fiqh Council of North America (1988). Son objectif est de former des comités de savants chargés de donner leur avis aux croyants en matière de fiqh (23). Il ne s'adresse pas aux seuls musulmans puisque les membres du FCNA ont également pour mission de répondre aux questions des non-musulmans, individus, institutions publiques, comme les ministères de la justice et de la défense, ou avocats (chargés par exemple de défendre les droits des minorités), journalistes, etc (24). Ces savants en religion doivent avoir les qualifications suivantes (similaires à maints égards à celles citées plus haut) : posséder une formation académique (sous-entendu une double formation en sciences laïques et en sciences religieuses) et un savoir linguistique (en arabe et en anglais), ne pas avoir de programme politique propre (l'autonomie est valorisée), respecter l'opinion des

(19) Cf. Aminah Mohammad-ArIf, ASSR, op. cit.

(20) La Jama'at-i Islami a été créée en Inde en 1941. Sur le rôle actif de cette organisation dans l'encadrement des fidèles aux États-Unis, cf. Aminah MoHAMmAD-ARIF, « Ilyâs et Maudûdi au pays des Yankees », op. cit.

(21) Titre honorifique utilisé pour un 'alim en Asie du Sud.

(22) Sur cette institution, cf. Barbara Metcalf, Islamic Revival in British India: Deoband, 1860-1900, Princeton, Princeton University Press, 1982, pp. 335-347.

(23) Jurisprudence; science du droit religieux.

(24) Yusuf Talal Delorenzo, op. cit., p. 68. 
juristes traditionnels sans toutefois y être lié de façon hermétique (ce qui suggère une porte ouverte à l'ijtihad (25)), avoir séjourné cinq ans minimum aux États-Unis (importance, accordée à la bonne connaissance du milieu ambiant) (26).

Par-delà les compétences requises, toutes ces organisations partagent la caractéristique commune d'utiliser largement les moyens technologiques comme Internet.

\section{Internet : entre individualisation et retour à l'auto-proclamation}

Ces organisations possèdent toutes leur site web que le croyant, indo-pakistanais ou non, peut aller consulter à sa guise, pour y poser ses questions sur les thèmes les plus variés. Certains sites sont spécialisés dans les fatwa. L'un des plus importants est sans doute islamonline.org. Il comporte un fatwa corner et fait appel aux bons offices de cyber counselors "habilités» (27) à prononcer des fatwa online. Aux États-Unis, les musulmans, indo-pakistanais notamment et jeunes en particulier (qui comptent de surcroît un nombre substantiel d'informaticiens), constituent une population spécialement encline à ce type de consultation religieuse. Internet encourage la transcendance des barrières ethniques. En effet, habituellement, les fidèles s'adressent de préférence à un leader religieux de leur propre groupe ethnico-national tandis que, sur le web, ils hésitent moins à solliciter des experts d'autre origine (pourvu qu'ils possèdent les qualifications requises). Soulignons enfin, avec Olivier Roy, que, aussi orthodoxes soient-elles, ces fatwa n'ont aucune valeur normative ou juridique. Elles ne valent que comme «avis» autorisé (28).

Les réponses sur un même sujet peuvent varier d'un site à l'autre et/ou être en contradiction avec celles de magazines islamiques. Prenons l'exemple de réponses trouvées l'une sur un site produit aux États-Unis, l'autre dans un magazine islamique également distribué en Amérique du Nord. Elles ont trait toutes deux au port de la barbe : l'une le recommande (tout en reconnaissant que ce n'est pas une obligation), l'autre insiste sur l'importance de l'intégration du fidèle dans sa société d'accueil :

The Prophet, peace be upon him, used to keep a beard, and his followers did the same, and he said : "trim your mustache (sic), and let your beard grow..." (Muslim (29)) That is why Imams Malek, Abu Hanefa, and Ahmed considered wearing a beard one of the obligations, but Imam Shafei said that it is undesirable to shave it, and to let it grow is Sunnah (but not obligation). And Allah knows best (30).

In truth, matters of wearing dresses along with the whole personal appearance, including shaving beards, are matters of costume for which a person should yield to what the societal environment accustomed to prefer one of the other of these costumes, he

(25) Effort personnel d'interprétations; travail des jurisconsultes dans la recherche de solutions juridiques.

(26) Yusuf Talal DE LoRENZo, op. cit.

(27) Les sites comportent généralement un curriculum vitæ du cyber counsellor consulté.

(28) Olivier Roy, L'islam mondialisé, Paris, Seuil, 2002, p. 83 (cf. Arch.122.45).

(29) Ibn Al-Hajjâj Muslim (mort en 875) est l'un des traditionnistes dont les Hadith sont les plus souvent cités.

(30) Réponse trouvée sur le site web du Fiqh Council of North America. 
should conform to them, otherwise his opposite attitude will be considered as isolation from the society (31).

Le caractère éminemment éclectique de ce nouveau type de consultation juridique n'en apparaît que plus manifeste, de même que ses effets immédiats, à savoir l'individualisation du religieux, un phénomène de plus en plus courant y compris dans les pays d'origine comme l'Inde et le Pakistan. Cette individualisation ne tient pas seulement au caractère volontaire de la démarche des croyants, mais aussi à la liberté de choix dont ils disposent. Certains peuvent s'attacher à l'avis d'un cyber counselor en particulier, mais bien d'autres sont tentés de multiplier leurs sources d'autorité et d'aller piocher des opinions ici et là. Le savoir ainsi vulgarisé et devenu accessible à tous, ou presque, ne repose plus sur un corpus délimité, placé sous le contrôle d'un groupe d'oulémas (32). Au contraire devenu quasi-inépuisable, du fait de la grande variété des sources possibles, il s'est "émancipé » de toute tutelle contraignante, voire coercitive.

Une autre conséquence de ces consultations d'un genre nouveau est la surenchère dans l'auto-proclamation, elle-même symptomatique de l'éclatement du paysage religieux. On ne manquera pas de souligner l'ironie de la situation : c'est précisément pour échapper aux imams auto-désignés dans les mosquées de l'immigration que les croyants ont cherché des sources alternatives d'autorité religieuse.

Ce phénomène d'autoproclamation est présent surtout chez les jeunes, certains, aux États-Unis comme ailleurs dans le monde, revendiquant un savoir supérieur à celui de leurs aînés en matière de religion. Cette prétention plonge ses racines non seulement dans la «vulgarisation d'un savoir religieux peu élaboré, grâce aux nouveaux supports » (33), mais aussi dans le sentiment de posséder une meilleure compréhension des textes islamiques et de la société américaine. Du point de vue religieux, les jeunes reprochent en effet à leurs parents d'appliquer les rites mécaniquement, sans effort de réflexion. Ce reproche est partiellement fondé, même si la première génération a elle aussi été obligée, du fait de sa situation migratoire à (re)penser son islam de façon plus explicite. Quoi qu'il en soit, aux États-Unis, les jeunes qui ont une fibre religieuse s'intéressent moins aux rites liés à une tradition particulière, la tradition sud-asiatique par exemple, qu'aux textes, qu'ils lisent, relisent et (ré)interprètent à la lumière de leurs besoins. Ainsi, à la différence des pratiques plus machinales observables dans le pays d'origine (y compris en Inde où les musulmans sont minoritaires (34)), en immigration la religion est appréhendée selon un mode plus rationnel. Cette démarche convainc les jeunes qu'ils sont les titulaires d'un savoir plus légitime que celui de leurs parents. À cela s'ajoute le fait que les jeunes, lorsqu'ils sont issus de la deuxième génération, sont (effectivement) plus familiarisés avec la société américaine, donc plus à même d'y saisir les besoins des musulmans. Cette conviction d'être détenteurs d'une double supériorité incite certains d'entre eux à se considérer comme les (seuls) leaders légitimes capables de guider les croyants et à s'autoproclamer savants en religion. Ces atouts cependant - accès au savoir, liberté de choix offerte par la multiplicité des sources,

(31) Réponse trouvée dans Resalah, mars 1994, p. 31.

(32) Olivier RoY, op. cit., p. 87.

(33) Ibid.

(34) La présence de l'islam en Inde est tellement ancienne que les musulmans indiens n'éprouvent pas nécessairement le même besoin de comprendre leur religion considérée comme faisant partie de l'évidence sociale et de l'habitus. 
autonomie vis-à-vis de structures religieuses pesantes, bagage «intellectuel» acquis grâce à la scolarisation, familiarité avec le milieu ambiant - ne les incitent pas pour autant à adopter une vision critique : nombre de ces jeunes privilégient les interprétations hermétiques des Textes et se contentent de réaffirmer des « vérités » immuables.

\section{Religion et politique : un leadership religieux dépolitisé en immigration?}

L'Inde et le Pakistan présentent des situations contrastées. Au Pakistan, les musulmans forment l'écrasante majorité de la population $(97 \%)$ et l'autorité religieuse y a été politisée et étroitement liée à l'État, en particulier depuis la politique d'islamisation du Général Zia ul Haq (1979) : zakat (35) obligatoire collectée par le gouvernement central, administration des waqfs (36) passée sous le contrôle de l'État, etc. En Inde, au contraire, les musulmans constituent une minorité (la plus importante des minorités, soit environ $13 \%$ ); toute intervention de l'État dans leurs affaires religieuses est considérée par eux comme une atteinte à leur liberté, inscrite dans la constitution, et comme une menace à leur statut de minorité religieuse disposant d'un statut propre. Le Parlement indien ne peut donc légiférer sur des questions de droit personnel touchant les musulmans. Néanmoins, les deux pays présentent des similitudes sur au moins un point : en Inde comme au Pakistan, oulémas et soufis peuvent jouer un rôle politique, les uns et les autres se présentant aux élections, endossant la fonction de leaders communautaires, etc. On y observe ainsi une confusion des genres entre autorité politique et autorité religieuse.

Aux États-Unis, la situation migratoire qui éloigne les croyants de toute structure religieuse établie liée à un gouvernement étranger, de même que l'absence de pressions directes de l'État sur le leadership religieux, inciteraient à penser qu'il n'existe pas, pour les musulmans, de lien entre autorité religieuse et autorité politique.

La différence avec l'Inde est moins radicale qu'avec le Pakistan, notamment parce qu'en Amérique comme en Inde, les musulmans sont minoritaires. Cela étant, alors qu'en Inde ils disposent d'un statut à part, aux États-Unis, où la liberté religieuse est protégée par la Constitution, les musulmans ne sont pas considérés comme une minorité bénéficiant d'une singularité juridique ou de droits « exceptionnels ». Pourtant, même aux États-Unis, la ligne de démarcation n'apparaît pas toujours très clairement entre autorité religieuse et autorité politique, en raison des chevauchements qui existent entre les leaderships religieux et communautaire (ce qui n'est pas sans rappeler la situation indienne). Nous avons vu en effet que certains leaders exercent plusieurs fonctions - un imam peut être porte-parole et se poser en leader communautaire -, ce qui participe d'une logique politique. Mais nous avons vu aussi que ce cas de figure demeure assez exceptionnel chez les

(35) Aumône légale.

(36) Fondation pieuse. 
Sud-Asiatiques, soucieux de départager les pouvoirs, alors qu'il est plus fréquemment observé chez les Noirs américains.

La complexité de la situation se manifeste également dans le fait qu'aux États-Unis ce sont des organisations distinctes, dans lesquelles les Sud-Asiatiques jouent un rôle notable, qui gèrent d'un côté les affaires religieuses de la communauté (comme l'ISNA, l'ICNA, le FCNA, etc.), et de l'autre les affaires politiques (comme l'American Muslim Alliance, l'American Muslim Council, ou encore le Muslim Public Affairs Council). Toutefois, si l'on prend l'exemple de l'American Muslim Council, une organisation qui a notamment endossé le rôle de lobby politique, on observe que ses dirigeants s'autorisent tout autant à écrire au Secrétaire général de l'ONU, Kofi Annan, une lettre dénonçant la répression politique dont sont victimes les musulmans aux États-Unis depuis le 11 septembre, ou à demander au Président Bush de ne pas attaquer l'Irak, qu'à organiser des séminaires sur la formation des imams. De même, des organisations comme l'ISNA ou l'ICNA, ou de simples imams, peuvent lancer des appels en faveur de la mobilisation politique des musulmans et encourager ces derniers à voter. Par ces actions, ces instances s'attribuent un rôle politique. Il est vrai que chacune d'elles s'efforce dans le même temps, officiellement au moins, de ne pas empiéter sur le champ de l'autre : l'AMC par exemple a recours, si besoin est, aux bons offices d'une autorité religieuse établie. Ainsi, en 2002, il a demandé à Taha Jaber al-Alwani, reconnu en Amérique du Nord par les croyants comme un expert en fiqh, de publier une fatwa (37) conseillant aux musulmans de participer aux élections américaines (lui faisant par là-même endosser une fonction politique).

Il apparaît au total que, sans se confondre systématiquement, les fonctions d'autorité religieuse et celle de leadership communautaire vont, à l'occasion et néanmoins, de pair. Rappelons que le contexte américain s'y prête particulièrement : tout en inscrivant dans la Constitution la séparation de l'Eglise et de l'État, les États-Unis confèrent au religieux une place fondamentale non seulement dans la vie privée, mais également, à maints égards, dans la vie publique et politique. Ce pays n'est donc pas un État laïque au sens français du terme. Il en résulte que le politique et le religieux sans nécessairement se trouver étroitement imbriqués chez les musulmans des États-Unis ne fonctionnent pas non plus de façon totalement séparée. Quant à la relation à l'État d'accueil, force est de constater que depuis le 11 septembre, la surveillance dont les musulmans font l'objet aux États-Unis, oblige leur leadership religieux à une auto-censure, en particulier dans leurs discours, ce qui tend à prouver que les instances locales exercent un contrôle politique, aussi indirect soit-il.

\section{Conclusion}

Le leadership religieux chez les musulmans des États-Unis a connu une évolution sensible. Afin de contrer l'émergence de leaders autoproclamés et de pallier le déficit initial en autorité religieuse, diverses initiatives ont été mises en œuvre: importation d'imams, création d'institutions et d'organisations visant à former de

(37) Avis religieux légal. 
futurs cadres religieux, etc. Ces formules ont connu des fortunes diverses, mais elles témoignent du dynamisme de la population musulmane, les Sud-Asiatiques en particulier avantagés par un haut niveau d'instruction. Elles sont également symptomatiques de l'importance conférée à l'héritage religieux par des immigrants déstabilisés par la situation diasporique, mais qui aspirent néanmoins à l'intégration et à une acceptation par la société-hôte.

Les sources d'autorité religieuse se sont diversifiées et multipliées, ce qui témoigne non seulement d'un éclatement de la scène religieuse et d'une individualisation du religieux mais également d'une liberté de choix offert aux croyants. Ce type de transformation s'observe aussi dans le reste du monde musulman, y compris dans le sous-continent indien, mais les États-Unis constituent un terreau particulièrement favorable en raison de l'extrême hétérogénéité de leur population musulmane et des moyens intellectuels, financiers et technologiques dont celle-ci dispose. Le fonctionnement démocratique de nombre des mosquées, où un partage des pouvoirs est assuré entre imam, conseil d'administration et fídèles, est l'une des autres spécificités remarquables de la redéfinition de l'autorité religieuse en contexte américain.

Depuis le 11 septembre, l'émergence d'un leadership religieux (tout autant que politique) " acceptable » aux yeux des autorités américaines est devenue un enjeu capital pour les musulmans, alors même que leurs activités sont placées sous étroite surveillance et que les Américains marquent un intérêt accru pour l'islam. Cela débouchera-t-il sur un remaniement du leadership musulman aux États-Unis ? Il est sans doute encore trop tôt pour le dire. Mais le 11 septembre a certainement été à l'origine de débats animés sur ces questions au sein de la communauté. Une nouvelle génération de leaders, mieux familiarisés avec la société américaine et plus soucieux de dépasser les clivages inter-ethniques (38), pourrait bien émerger.

\author{
Aminah MOHAMMAD-ARIF \\ Centre d'Études de l'Inde et de l'Asie du Sud \\ CNRS
}

(38) Depuis le 11 septembre les échanges entre diverses organisations religieuses et politiques représentant des musulmans d'affiliation ethnique différente se sont multipliés. Il est par exemple remarquable que les Sud-Asiatiques, qui jusqu'à présent avaient «boudé » les Afro-Américains, sont désormais plus enclins à les associer à leurs activités et surtout à les solliciter sur les questions de discrimination. En effet, les Sud-Asiatiques ont à présent pris conscience de la signification de l'ethnic profiling, dont les Afro-Américains ont eu à souffrir depuis des décennies, voire des siècles, et espèrent bénéficier de l'expérience de ces derniers dans la lutte contre la discrimination, dont tous les musulmans, par-delà l'appartenance ethnique (mais les immigrants plus que les «indigènes »), sont aujourd'hui les victimes. 
Résumé

L'autorité religieuse chez les musulmans des États-Unis, les Indo-Pakistanais en particulier, a connu au fil des ans une évolution sensible, les immigrants ayant tenté diverses formules destinées à pallier le déficit initial en la matière et à contrer la montée de leaders autoproclamés. Les sources d'autorité religieuse se sont diversifiées et multipliées, ce qui témoigne non seulement d'un éclatement de la scène religieuse et d'une individualisation du religieux mais également d'une liberté de choix aux États-Unis offerte aux croyants. Depuis le 11 septembre 2001, l'émergence d'un leadership religieux "acceptable " aux yeux des autorités américaines est devenue un enjeu crucial pour les musulmans alors même que leurs activités sont placées sous étroite surveillance. Aussi la question de l'autorité religieuse est-elle aujourd'hui un sujet majeur de débats au sein de cette population.

\section{Abstract}

Religious authority among Muslims in the United States, South Asians in particular, has significantly evolved over the years : they have tried several methods in order to compensate for the initial lack of authority ant to counter the rise of self-proclaimed leaders. There has been a diversification and multiplication of the sources of religious authority, this showing not only the fragmentation of the religious arena and the individualization of religion but also a freedom of choice available to the believers in the United States. Since September $11^{\text {th }}$, the emergence of a religious leadership acceptable to the American authorities has become all the more crucial as the activities of the Muslims have been put under close scrutiny. As a matter of fact, the question of religious authority has generated animated debates within the community.

\section{Resumen}

La autoridad religiosa entre los musulmanes de los Estados unidos, los Indo-paquistanies en particular, ha conocido con los años una evolución sensible, ya que los inmigrantes han intentado diversas fórmulas destinadas a paliar el déficit inicial en materia y a oponerse al ascenso de líderes auto-proclamados. Las fuentes de autoridad religiosa se han diversificado y multiplicado, lo que da cuenta no sólo de una fragmentación de la escena religiosa y de una individualización de lo religioso, sino también de una libertad de elección que en los Estados Unidos se ofrece a los creyentes. Desde el 11 de septiembre de 2001, la emergencia de un liderazgo religioso "aceptable" para las autoridades americanas, se ha vuelto una apuesta crucial para los musulmanes, en el momento en que sus actividades son puestas bajo una estrecha vigilancia. Así, la cuestión de la autoridad religiosa es hoy un tema mayor de debate en esta población. 
